\title{
Síndrome de May-Thurner como diagnóstico diferencial en trombosis a repetición
}

\section{May-Thurner syndrome as a differential diagnosis in recurrent thrombosis}

\author{
Eugenia lucía Saldarriaga, Miguel Vinasco, Diego Alejandro Jaimes \\ - Bogotá, D.C. (Colombia)
}

\section{Resumen}

El síndrome de May-Thurner es una anomalía anatómica que genera insuficiencia venosa y episodios trombóticos recurrentes de los miembros inferiores.

Se presenta el caso de una paciente de 29 años, con cuadro de trombosis venosas profundas en miembros inferiores a repetición desde los 20 años, sin factores de riesgo identificados, en quien se realizan estudios de extensión para neoplasia y trombofilia con resultados negativos, además de estudios para patología reumatológica con anticuerpos antinucleares (ANAS) positivo a títulos bajos como único hallazgo, con lo que se hace diagnóstico de enfermedad del tejido conectivo no diferenciado y se instaura manejo con anticoagulación plena; sin embargo, ante la persistencia de eventos trombóticos se realiza angio-TAC, la cual reporta obstrucción de la vena ilíaca izquierda, por lo cual se realiza una venografía más cavografía documentándose obstrucción de la vena ilíaca externa izquierda y la común estableciéndose el diagnóstico de síndrome de May-Thurner, el cual debe considerarse dentro de los diagnósticos diferenciales de los episodios trombóticos recurrentes. (Acta Med Colomb 2016; 41: 67-70).

Palabras clave: síndrome de compresión ilíaco, síndrome de May-Thurner, síndrome de Cockett, síndrome postrombótico, trombosis venosa profunda.

\begin{abstract}
The May-Thurner syndrome is an anatomic abnormality generating venous insufficiency and recurrent thrombotic episodes of lower limbs.

The case of a 29 years old patient with clinical picture of recurrent deep venous thrombosis in lower limbs since the age of 20 years is presented. No risk factors were identified; extension studies for neoplasia and thrombophilia were performed with negative results, as well as studies for rheumatic disease with positive anti-nuclear antibodies (ANAs) at low titers as unique finding, being diagnosed as undifferentiated connective tissue disease and management with full anticoagulation is established. However, at the persistence of thrombotic events, an angio-CT which reports obstruction of the left iliac vein and the common vein is performed whereby a venography and cavography are done establishing the diagnosis of May Thurner syndrome, which should be considered within the differential diagnosis of recurrent thrombotic events. (Acta Med Colomb 2016; 41: 67-70).
\end{abstract}

Keywords: iliac compression syndrome, May-Thurner syndrome, Cockett syndrome, postthrombotic syndrome, deep venous thrombosis.
Dra. Eugenia Lucía Saldarriaga Cardeño: Internista. Residente de Reumatología; Dr. Miguel Vinasco: Residente de Medicina Interna, Facultad de Medicina, Universidad de La Sabana, Chía (Cundinamarca); Dr. Diego Alejandro Jaimes Fernández: Internista, Reumatólogo, Facultad de Medicina, Universidad de La Sabana, Hospital Universitario de La Samaritana. Bogotá, D.C. (Colombia).

Correspondencia: Dr. Diego Jaimes. Bogotá, D.C. (Colombia).

E-mail: diegojf@unisabana.edu.co

Recibido: 26/02/2015 Aceptado: 23/02/2016

\section{Introducción}

El síndrome de May-Thurner, Cockett o síndrome de compresión iliocava, es aquella alteración anatómica, compresiva o endoluminal de la vena ilíaca común izquierda, que produce obstrucción y/o trombosis venosa iliofemoral.

El grado de severidad de las manifestaciones clínicas de este síndrome, depende de la eficiencia de la circulación colateral de la pelvis para lograr llevar el retorno venoso desde la extremidad inferior hacia la vena cava inferior, dando la posibilidad de encontrar pacientes que aunque tengan evidente malformación endoluminal o compresión extrínseca su efecto compensatorio es mayor evitando así eventos trombóticos. 
Los eventos trombóticos ante positividad de laboratorios inmunológicos a títulos bajos no siempre corresponden a entidad autoinmune.

El síndrome de May-Thurner es una anormalidad anatómica subvalorada la cual debe evaluarse ante eventos no claros de trombosis, de allí la importancia de este caso.

\section{Presentación del caso}

Mujer de 29 años, natural de Cundinamarca, con cuadro clínico de nueve años de evolución de múltiples trombosis venosas profundas en miembros inferiores seis en total, comprometiendo vasos venosos iliofemoropoplíteos izquierdos en forma repetitiva.

Sin ningún otro hallazgos al examen físico, sin antecedente de cáncer ni cirugías previas en ninguno de los eventos, por lo cual se decidió realizar estudios de extensión para neoplasia (Tabla 1) descartándose la misma, estudios negativos para trombofilia, dentro del perfil inmunológico de 2005 se evidenció ANAS positivos 1:80 patrón moteado los cuales persisten positivos en el tiempo, en 2011 anti-Sm positivo; sin embargo, al no cumplir ningún criterio de patología reumatológica se realizó el diagnóstico de enfermedad del tejido conectivo no diferenciado y se inició manejo con anticoagulación plena.

Debido a eventos repetitivos se considera en 2011 realización de angiotomografía abdominopélvica la cual reportó obstrucción de la vena ilíaca izquierda, várices pélvicas con circulación colateral hipogástrica, se realizó nuevo perfil autoinmune con ANAS presentando nuevamente títulos bajos y patrón moteado, ENAS negativos, debido a estos hallazgos se realizó flebografía más iliocavografía (Figuras 1 y 2 ) documentándose obstrucción de la vena ilíaca externa

Tabla 1. Resultados de laboratorio.

\begin{tabular}{|c|c|c|c|}
\hline 2005 & 2009 & 2011 & 2014 \\
\hline Antifosfolípido negativo & Mutación del factor $\mathrm{V}$ negativo & Antifosfolípido negativo & Complemento sérico fracción C3 $188.79 \mathrm{mg} / \mathrm{dl}$ \\
\hline Anticoagulante lúpico negativo & Proteína $\mathrm{S}$ y $\mathrm{C}$ dentro de rangos normales & Anticoagulante lúpico negativo & Complemento sérico fracción C4 $27.7 \mathrm{mg} / \mathrm{dl}$ \\
\hline Anticardiolipina negativo & Protocolo plaqueta pegajosa negativo & Anticardiolipina negativo & AntiSCL-70 8.17 negativo \\
\hline B2glicoproteínas negativos & Anti-Sm 21 positivo & B2glicoproteinas negativos & ENAS negativo \\
\hline ENAS negativo & Anti-Ro negativo & ENAS negativo & ANAS negativo \\
\hline ANAS 1:80 patrón moteado & Anti-La negativo & ANAS 1:80 patrón moteado & Anti-DNA negativo \\
\hline Tomografía toracoabdominal negativo & Anti-RNP negativo & & Anti-fosfolípido negativo \\
\hline Ca19-9 negativo & ANAS 1:80 patrón moteado & & Factor VIII normal \\
\hline Antígeno-carcionoembrionario & & & Antitrombina III negativo \\
\hline
\end{tabular}

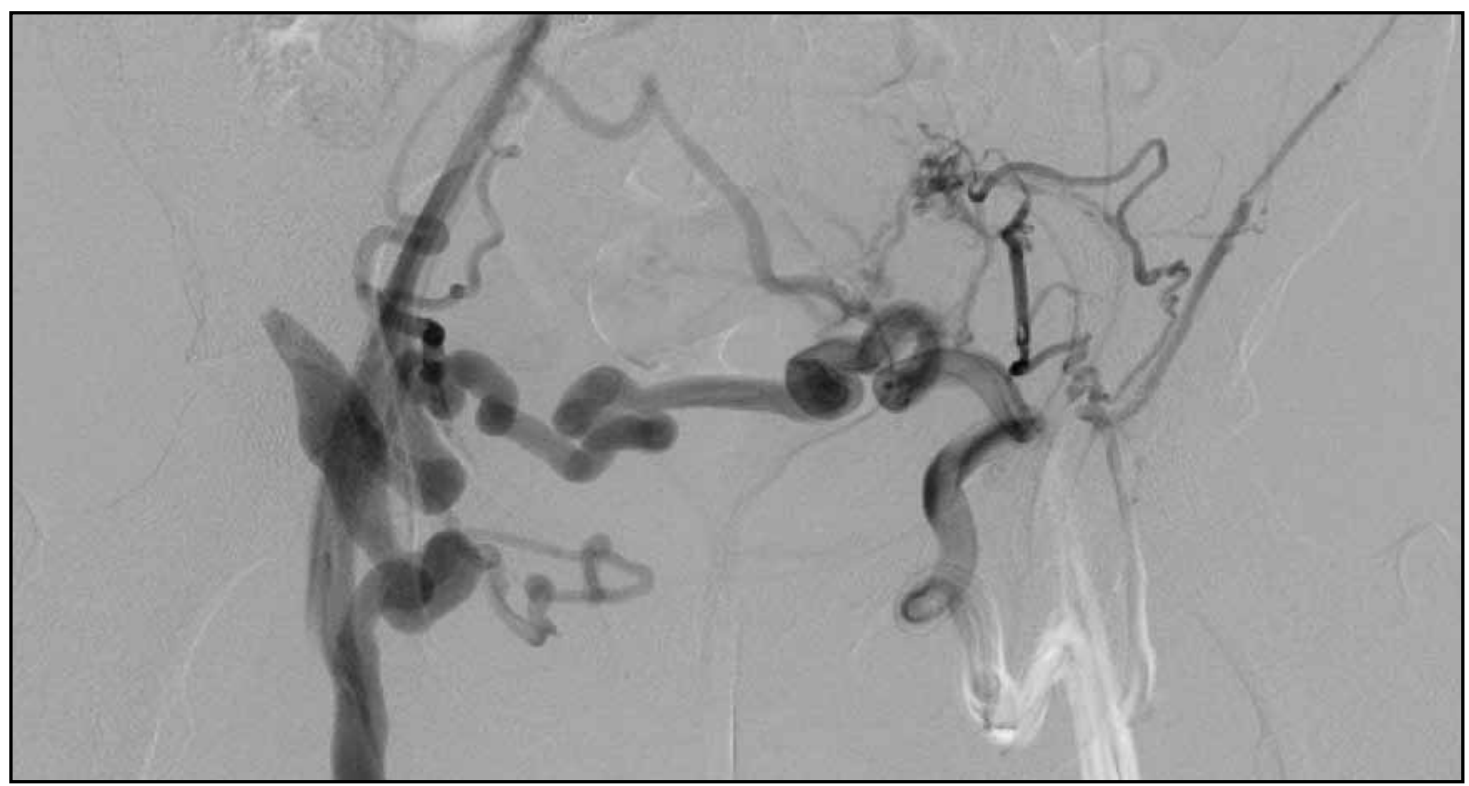

Figura 1 . Flebografía. 


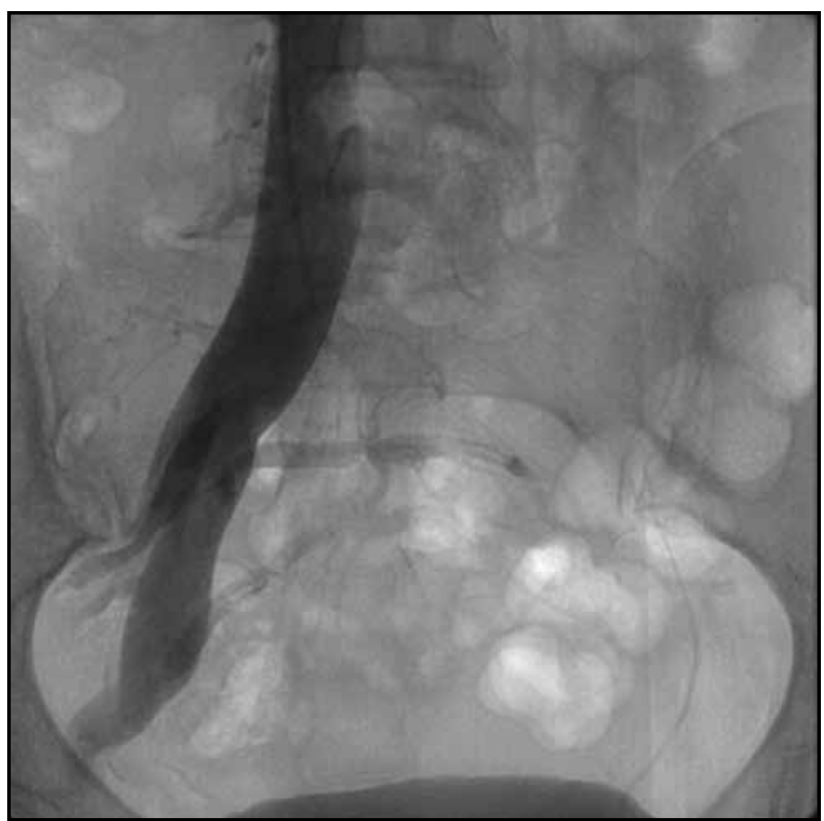

Figura 2. Iliocavografía.

izquierda y la común denominado síndrome de Cockett, anormalidad anatómica la cual desencadenó estos eventos por lo cual se propone manejo endovascular con stent, en la actualidad perfil autoinmune negativo.

Es importante recalcar que no todo lo positivo en el perfil inmunológico es autoinmune, ante eventos trombóticos a repetición es importante evaluar anormalidades anatómicas, de allí la importancia de este caso.

\section{Discusión}

El síndrome de compresión de la vena ilíaca, síndrome iliocavo, de Cockett o de May-Thurner es una condición en la cual la vena ilíaca común izquierda se encuentra comprimida a su paso entre la arteria ilíaca común derecha y la columna a la altura del cuerpo vertebral de la quinta vértebra lumbar o el promontorio del sacro justo antes de la unión iliocava (1-3) con la consecuente aparición de trombosis venosa profunda en la extremidad inferior izquierda, lo cual puede llevar a insuficiencia venosa crónica o trombosis repetidas a largo plazo (3).

La primera descripción fue realizada por Virchow en 1851 quien documenta una frecuencia cinco veces mayor de trombosis del lado izquierdo con respecto al derecho, posteriormente McMurrich en 1908 describe adhesiones y edema en las venas ilíacas de 35 cadáveres, de los cuales 32 comprometían la vena ilíaca izquierda, pero sólo es hasta el año 1957 cuando May y Thurner realizan la descripción anatómica precisa tras examinar 430 cadáveres y describen lo que denominan como la transformación cicatricial esclerótica causada por inflamación perivenosa secundaria a la compresión (4). Las características clínicas fueron publicadas por Cockett y Thomas en 1965 y 1967 con una serie de 57 pacientes (5).
A la fecha se sabe que si bien la superposición de los vasos es anatómicamente normal, en algunos pacientes el efecto que genera la compresión posterior de la vena con el cuerpo vertebral y la compresión anterior con la arteria ilíaca induce dos mecanismos, el primero la obstrucción mecánica al flujo y el segundo la hiperplasia intimal y la formación de membranas o bandas de elastina y colágeno $(1,6)$ como consecuencia de la transmisión de la fuerza pulsátil crónica desde la arteria que ocasiona fricción entre las paredes de la vena (7). La disminución de la luz vascular, con el compromiso del drenaje venoso conduce a la presencia de edema unilateral, aparición de circulación colateral, trombosis aguda de la vena ilíaca común o incluso ruptura espontánea de la misma (1, 7-9). La presencia de esta variación anatómica asociada a factores como inmovilidad, embarazo, anticoncepción oral, trauma o cáncer puede incrementar el riesgo de desarrollar un episodio de trombosis venosa (10).

Su prevalencia real es desconocida, es más frecuente en mujeres entre los 20-40 años y se considera que esta entidad es frecuentemente subdiagnósticada (1), se ha reportado una prevalencia de $22-24 \%$ de este síndrome en un análisis retrospectivo por medio de tomografía axial $(6,11)$; sin embargo, de forma llamativa a pesar de la frecuencia de la variación anatómica, la prevalencia del síndrome de May Thurner asociado a trombosis venosa profunda es sorpresivamente bajo, ocurriendo en 2-3\% de todas los episodios de trombosis de miembros inferiores $(6,12)$, lo que hace pensar aún más en la presencia de subdiagnóstico.

La manifestación más frecuente es la trombosis venosa profunda; sin embargo, puede presentarse con hallazgos sugestivos de insuficiencia venosa crónica con dolor, edema, tumefacción, claudicación venosa e insuficiencia venosa sin trombosis, o menos frecuentemente con várices, úlceras venosas y complicaciones como ruptura espontánea del vaso, tromboembolismo pulmonar o flegmasia cerúlea dolens (1, 9, 13). Los estadios clínicos fueron descritos por Kim y colaboradores e incluyen estadio I compresión asintomática, estadio II aparición de "Spur o espolones venosos" y estadio III trombosis de la vena ilíaca izquierda (14).

Diferentes modalidades de imágenes han sido utilizadas como resonancia magnética, tomografía axial y ultrasonografía intravascular; sin embargo, la venografía ilíaca realizada por vía femoral es la prueba diagnóstica de elección debido a que ésta puede demostrar la compresión y a que la medición de los gradientes de presión puede realizarse para confirmar los hallazgos (7), para hacer el diagnóstico de una estenosis significativa la diferencia de presiones entre las dos venas ilíacas debe ser mayor de $2 \mathrm{mmHg}$ en reposo o de $3 \mathrm{mmHg}$ durante actividad, otros autores recomiendan la medición de la presión proximal y retrógrada como prueba de mayor validez (13). El doppler color es una prueba simple y no invasiva pero el estudio de los vasos pélvicos es técnicamente difícil principalmente en pacientes obesos o si se presenta interposición de gas intestinal lo que limita su uso (13). 
El tratamiento endovascular de la enfermedad ha emergido durante los últimos años como la terapia de elección, siendo el implante de stents la estrategia de elección debido a la continua compresión de la vena, hasta el momento sólo hay algunos reportes con pocos pacientes y diseños metodológicos inadecuados que reportan buena respuesta clínica y alivio de los síntomas (15), la baja frecuencia de la enfermedad limita la realización de estudios más grandes y con diseños metodológicos adecuados. Posterior al implante de éstos, se requiere la anticoagulación sostenida por un periodo de mínimo seis meses, la presencia de flujos venosos normales dos años después del implante del stent son encontrados según los reportes de algunos autores en 95-100\% de los casos (12).

En resumen, ante la presencia de pacientes con trombosis venosa profunda recurrente no explicada o edema en el miembro inferior izquierdo asociado a signos de insuficiencia venosa crónica especialmente en mujeres jóvenes, debe sospecharse la presencia de esta alteración anatómica y confirmarse con venografía ilíaca.

\section{Declaración de fuentes de financiación y posibles conflictos de interés}

Fuentes de financiación: Recursos propios de los autores Conflicto de Intereses: Ninguno

\section{Referencias}

1. Martín, A.G., et al. Síndrome de Cockett o de May-Thurner o síndrome de compresión de la vena ilíaca. Radiología. 2014.56 (5): p. e5-e8.

2. Oteros, F.R., et al. May-Thurner syndrome and surgery for scoliosis. Radiología. 2008. 50 (3): p. 245.

3. Butros, S.R., et al. Venous compression syndromes: clinical features, imaging findings and management. British J Radiol. 2013.86 (1030): p. 20130284.

4. May, R. and J. Thurner. The cause of the predominantly sinistral occurrence of thrombosis of the pelvic veins. Angiology. 1957.8 (5): p. 419-27.

5. Menon, R. Nonthrombotic Iliac Vein Lesion (May-Thurner Syndrome), in Chronic Venous Disorders of the Lower Limbs. 2015, Springer India. p. 223-228.

6. Kalu, S., et al. May-Thurner syndrome: a case report and review of the literature. Case repo vasc medic. 2013. 2013.

7. Cil, B.E., et al. Case 76: May-Thurner Syndrome. Radiology. 2004. 233 (2): p 361-365.

8. Loukas, M., et al. A case of May-Thurner syndrome. Folia morpholo. 2008.67 (3): p. 214-217.

9. Gordillo-Escobar, E., et al. Síndrome de May-Thurner: causa infrecuente de rotura espontánea de vena ilíaca izquierda. Med inten. 2012.36 (3): p. 239-240.

10. McDermott, S., et al. May-Thurner syndrome: can it be diagnosed by a single MR venography study. Diagn Interv Radiol. 2013.19 (1): p. 44-48.

11. Kibbe, M.R., et al. Iliac vein compression in an asymptomatic patient population. $J$ vasc surg. 2004.39 (5): p. 937-943.

12. Peters, M., et al. May-Thurner syndrome: a not so uncommon cause of a common condition. Proceedings (Baylor University. Medical Center). 2012. 25 (3): p. 231

13. Ahmed, H.K. and K.D. Hagspiel. Intravascular ultrasonographic findings in May-Thurner syndrome (iliac vein compression syndrome). J ultras medic. 2001. 20 (3): p. 251-256.

14. Ibrahim, W., et al. Endovascular Management of May-Thurner Syndrome. Annals vasc disea. 2012.5 (2): p. 217.

15. Canales, J.F. and Z. Krajcer. Intravascular ultrasound guidance in treating May-Thurner syndrome. Texas Heart Instit J. 2010.37 (4): p. 496. 